\title{
Andre Bogart Szabo Art Images
}

\author{
Andre Bogart Szabo
}

\section{Artist's Statement}

As a visual artist, I am fascinated with the inherent beauty of physical materials themselves, unmediated and unrefined. These works on canvas feature repurposed, foraged, and composted objects; a material vocabulary that follows no hierarchy. My paintings challenge the rhetoric of purity, refinement, and expressionism bound to painting by means of a 'soil over oil' approach. Tapping natural materials as alternatives to brush and gesture, my paintings have a sense of immediacy that directly engage the social, cultural, and ecological concerns of the region from which they are sourced. Just as a responsible hunter uses every piece of their kill as functional material, so do I process the physical world so nothing goes to waste.

In relation to Nietzsche, my works on canvas celebrate concepts presented in 'The Birth of Tragedy,' especially the duality between Apollo and Dionysus (order/disorder, rational logic/amorous frenzy...consider Dionysus as presented in Euripedes' The Bacchae). In addition, my work is inspired in part by the 'excremental philosophy' of Georges Bataille, a philosopher who wrote extensively on Nietzsche.

Andre Bogart Szabo maintains an artist studio in Red Hook, Brooklyn. He was born in Washington, DC in 1990. In 2012, he received his BA from Emerson College's Visual Media Arts department. Bogart Szabo is the recipient of various awards, scholarships, and residencies. In 2021, he will be included in New American Paintings Issue \#154. In 2020, he has been awarded numerous grants that include: Foundation for Contemporary Arts, Rauschenberg Grant Fund, Washington Project for the Arts, and Artists Fellowship, Inc. In the spring of 2019, he was invited to participate in the Bronx Museum of the Arts AIM Fellowship Program. 


\section{Image List}

01 Ghost Ranch (Still Life XII), 2021, Mixed media on canvas, 38 x 32 inches

02 Holy Dirt (New Mexico), 2021, Mixed media on canvas, 41 x 31 inches

03 Nothing Goes to Waste (Trophy II), 2021, Mixed media on canvas, 36 x 24 inches

04 Nothing Goes to Waste (Trophy II), detail

05 Shore Wash (Landscape V), 2020, Mixed media on canvas, 84 x 84 inches

06 Coffee Pourover (Landscape II), 2020, Mixed media on canvas, 84 x 54 inches

07 Catskill Smiling Deer (Trophy I), 2020, Mixed media on canvas, 84 x 54 inches

08 Valley of Fire, 2021, Mixed media on canvas, $32 \times 28$ inches 


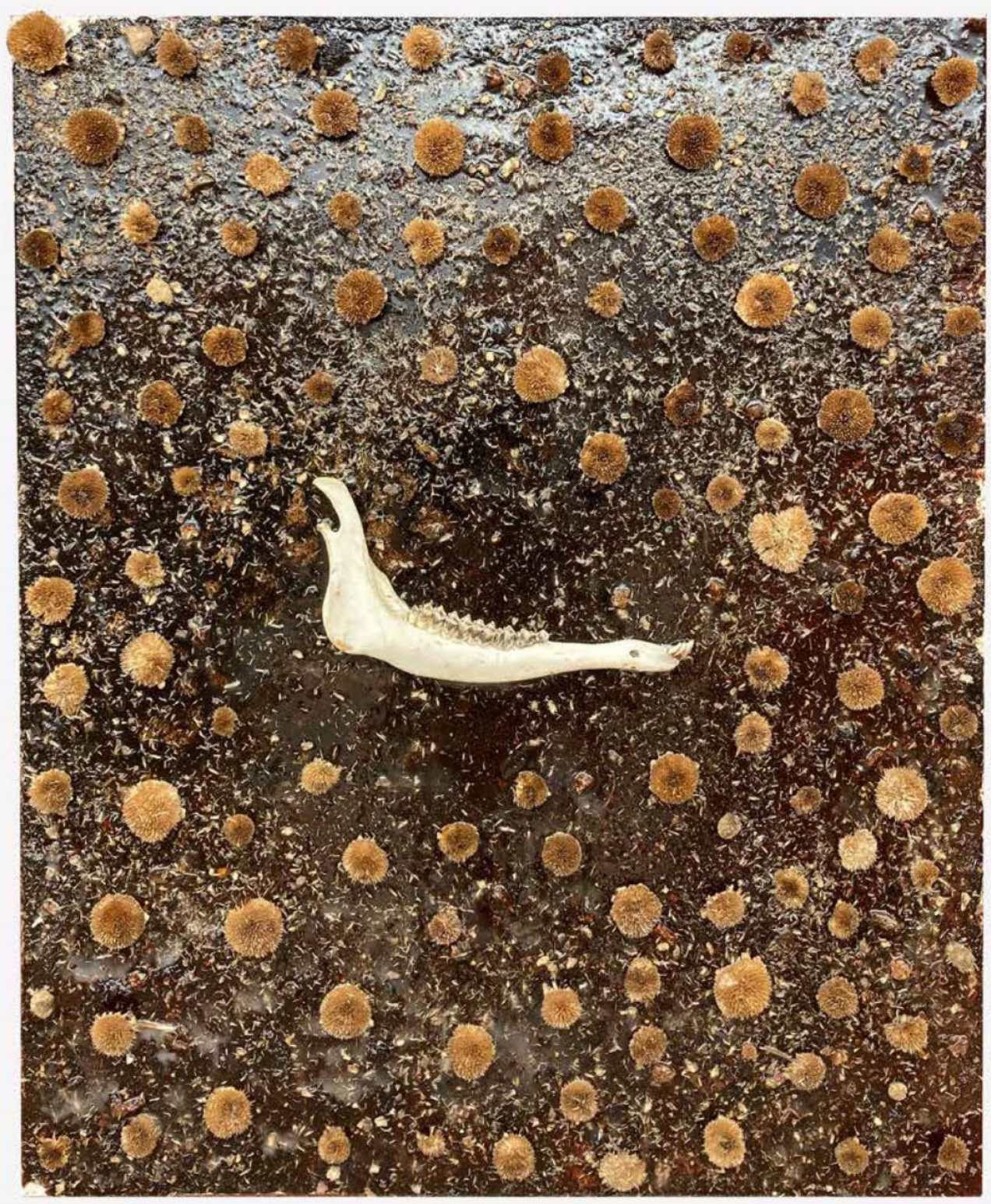




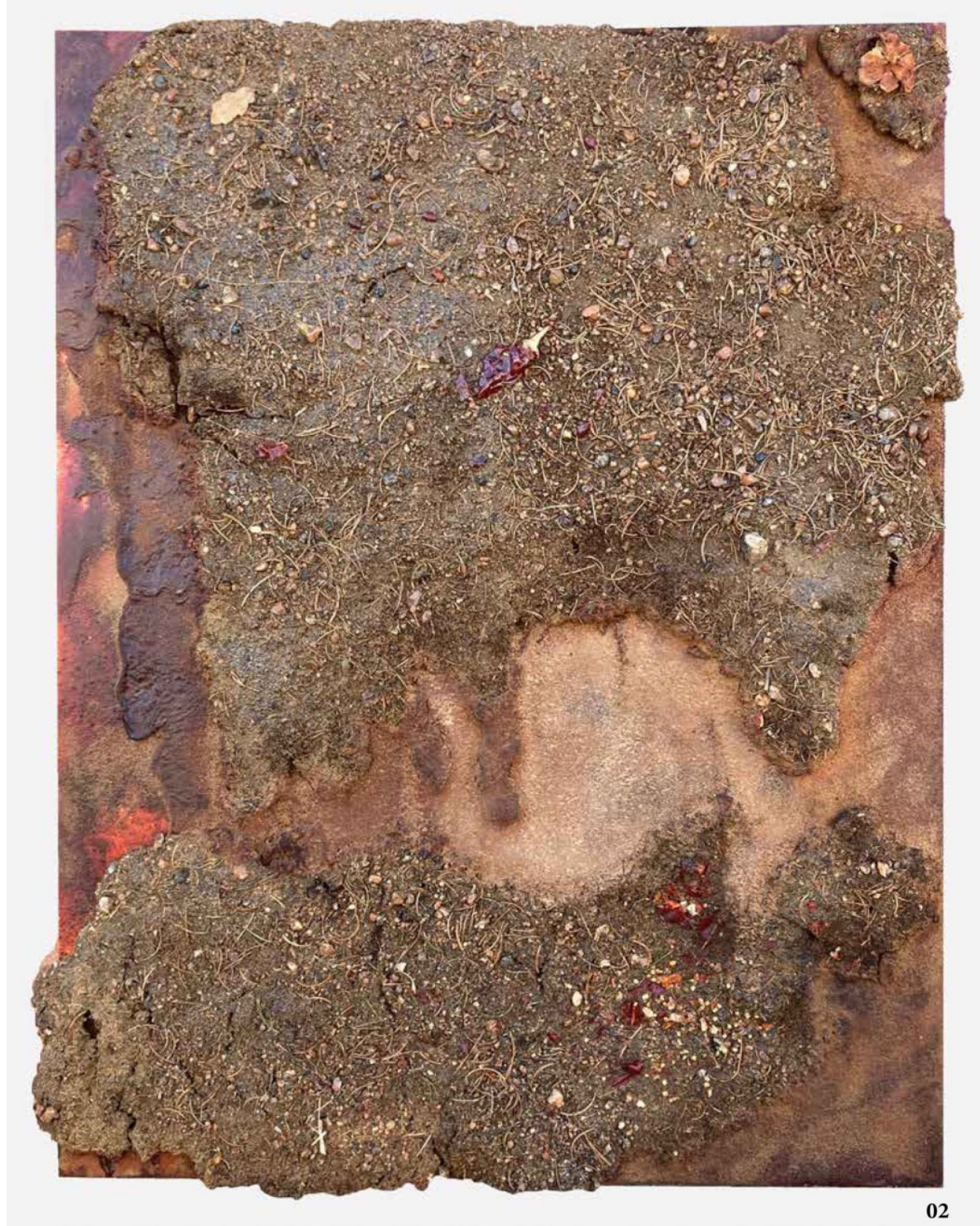




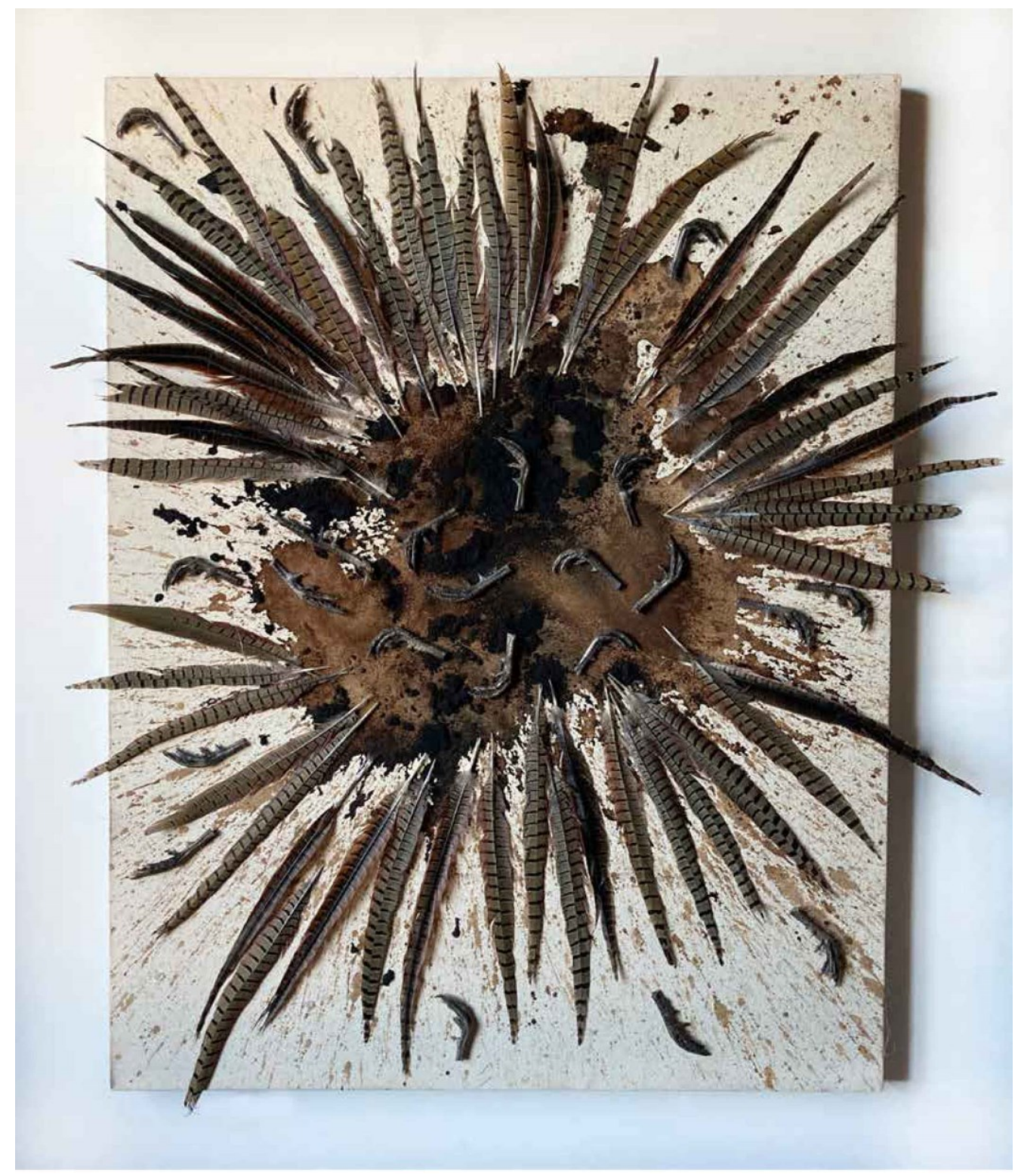


96 Andre Bogart Szabo Art Images

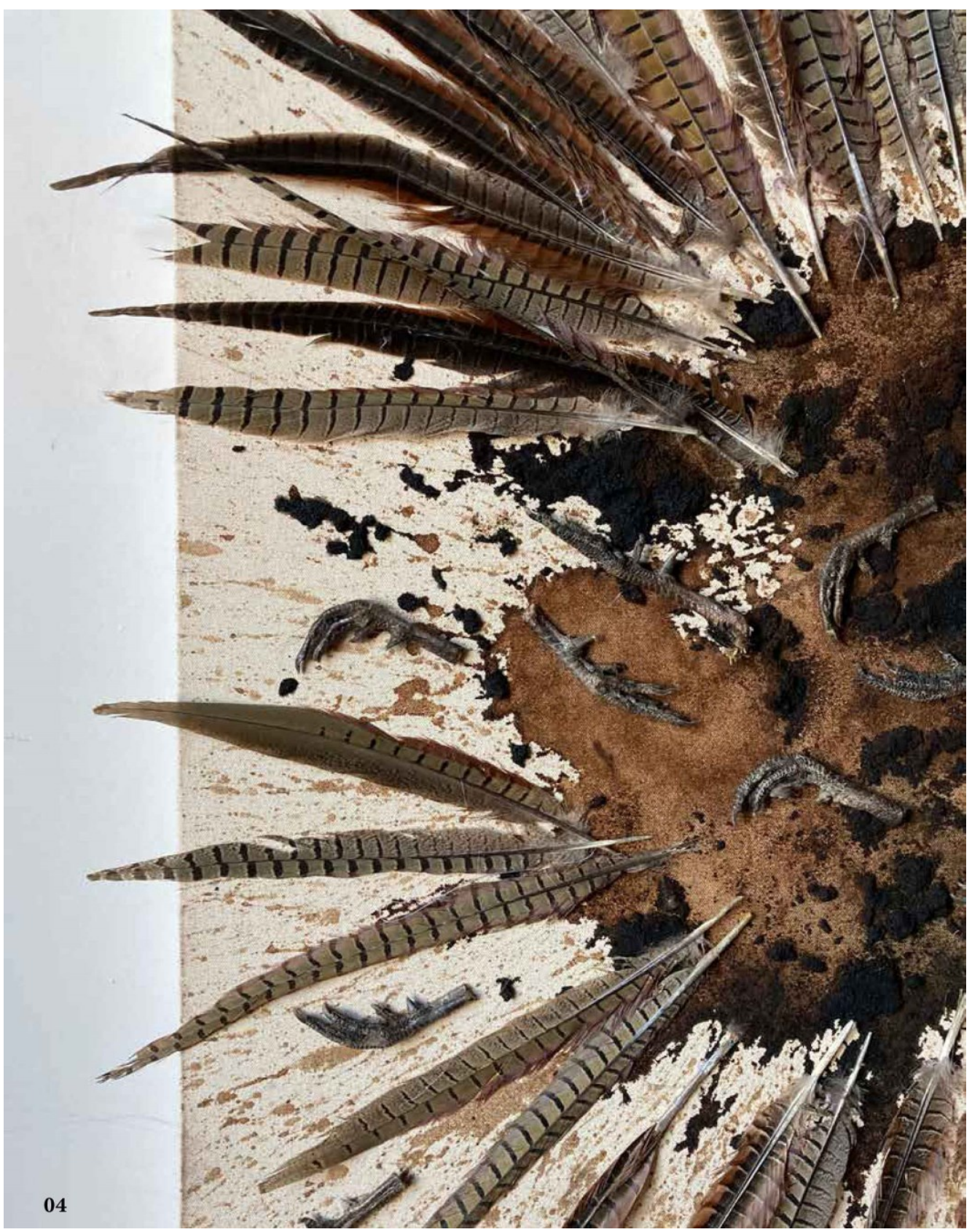




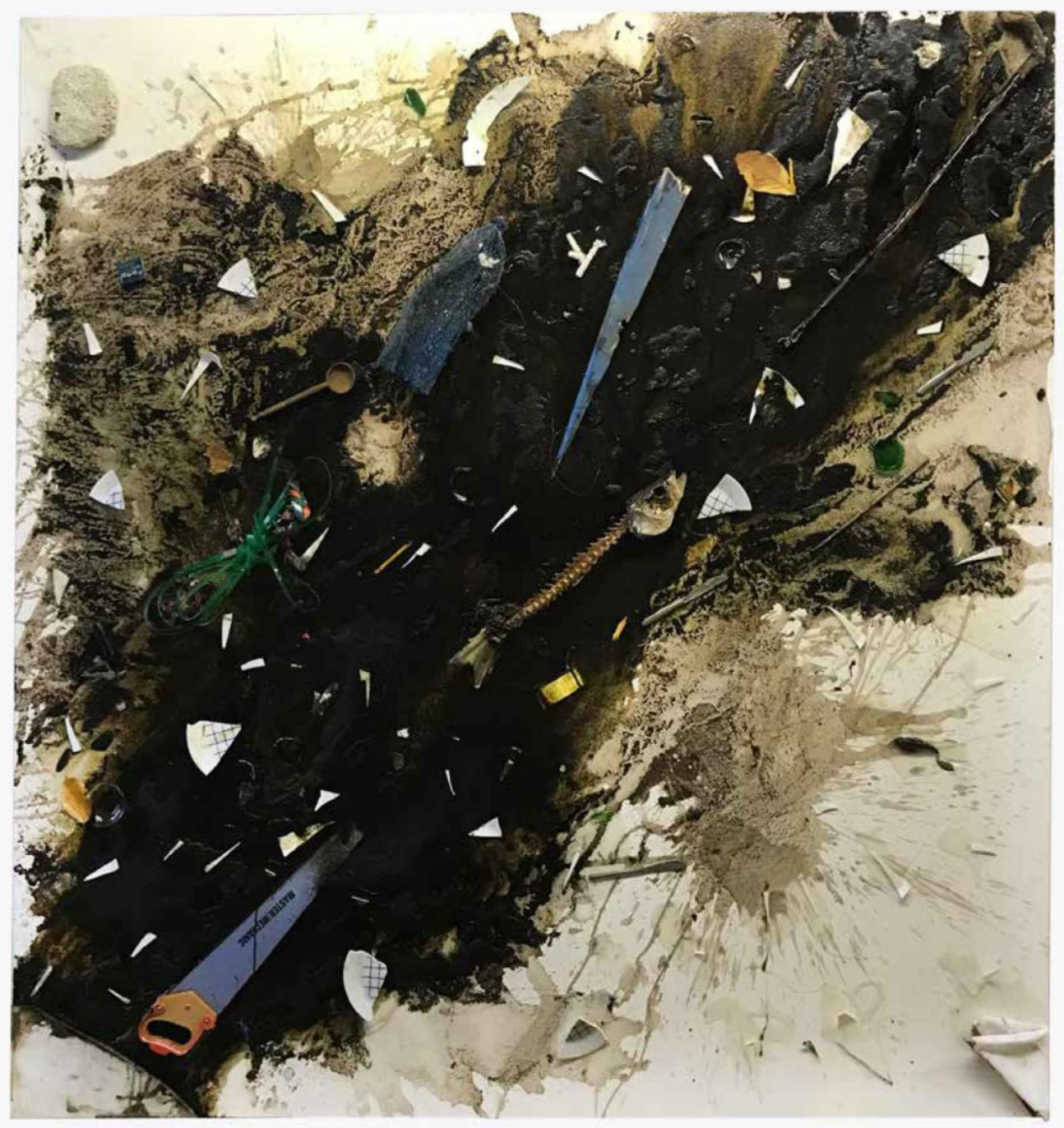




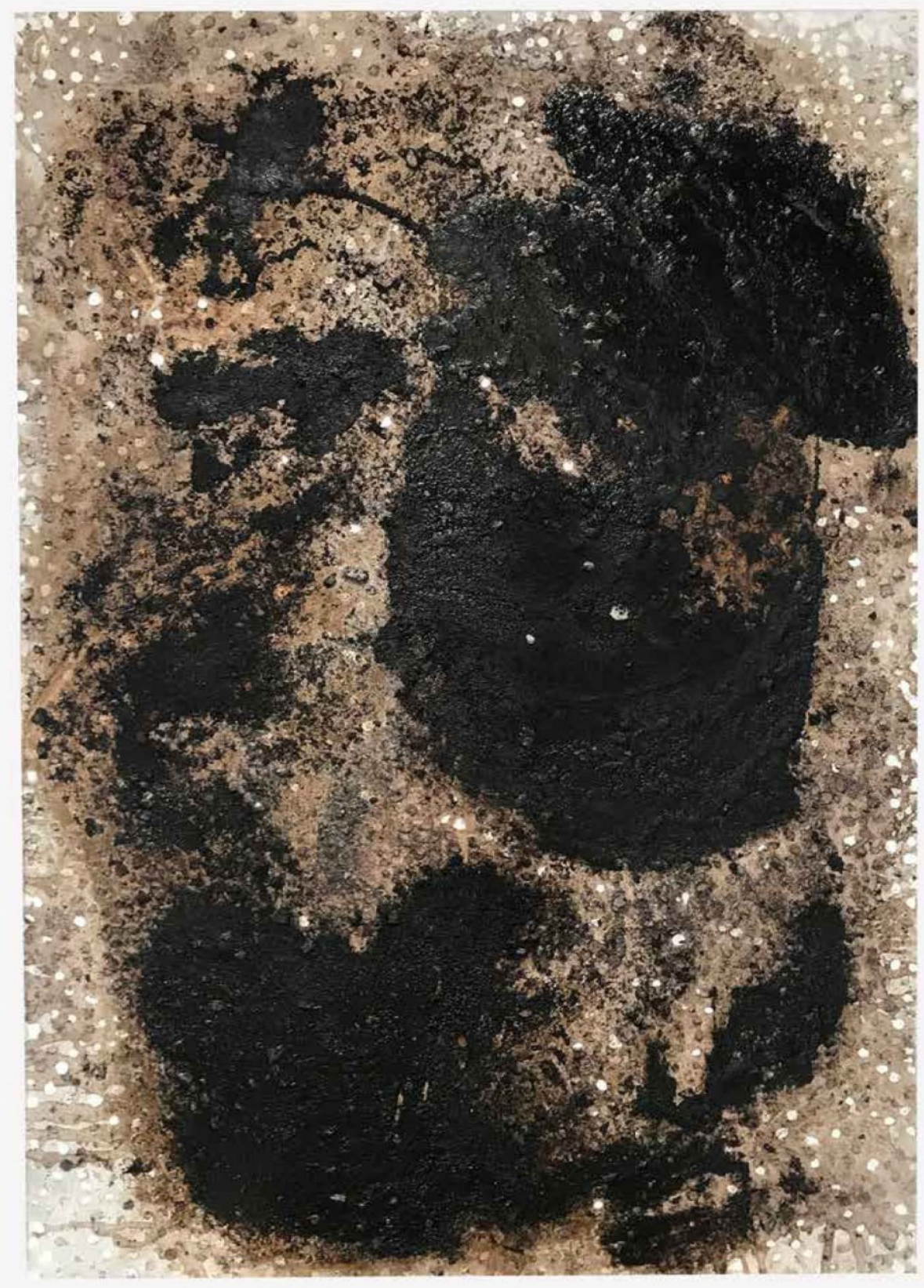




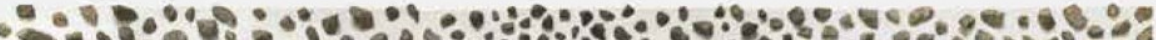

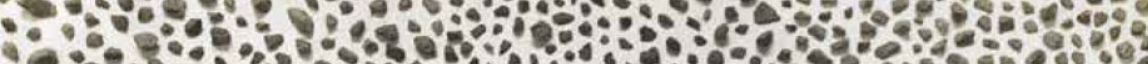

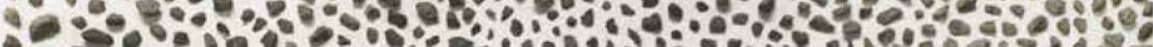

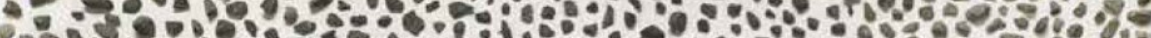

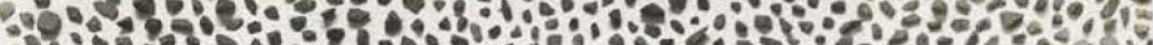
H:

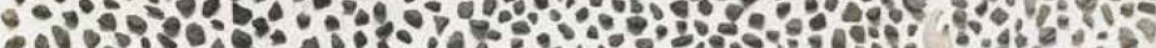

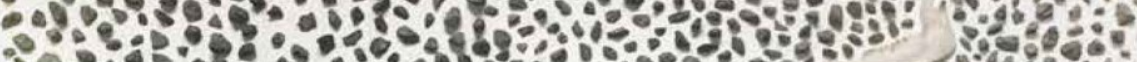
:-

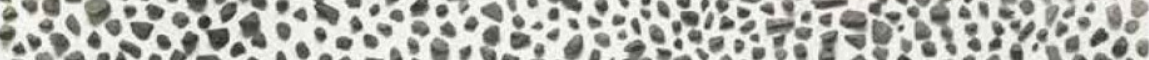
\% - -28 -

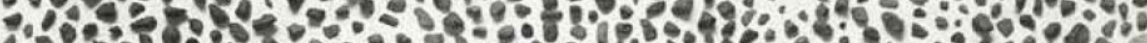

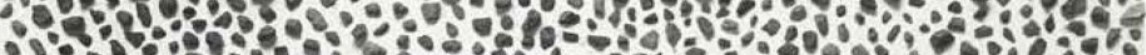
-5.

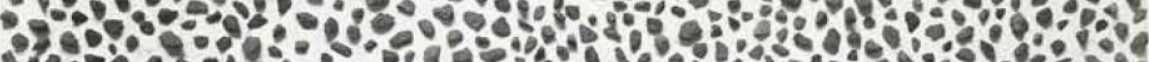

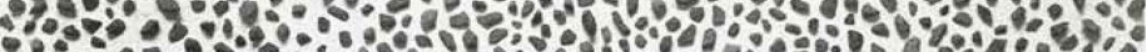

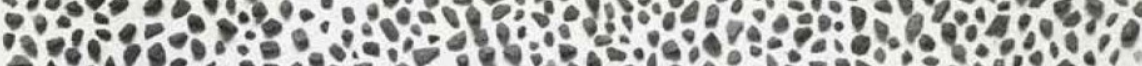

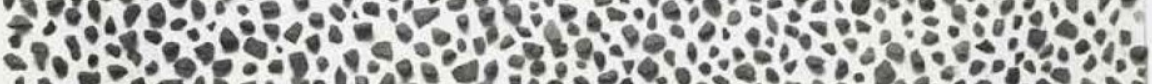

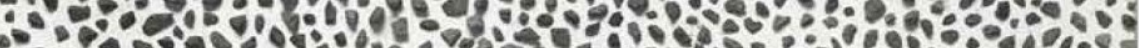

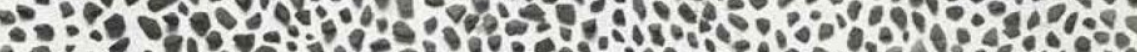

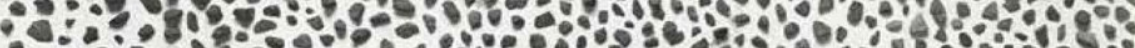

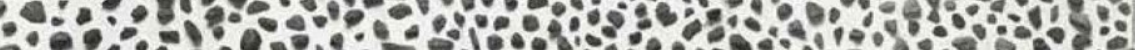

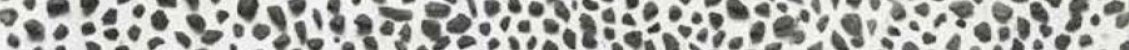
4. - -

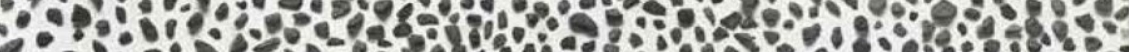

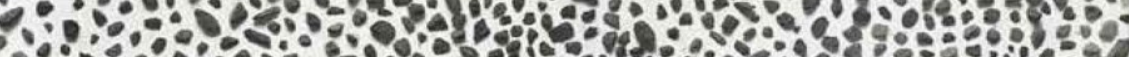

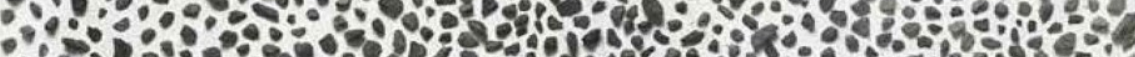

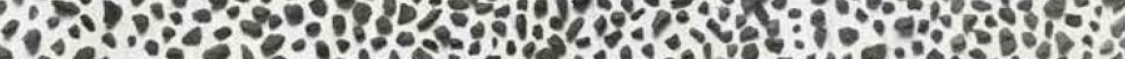

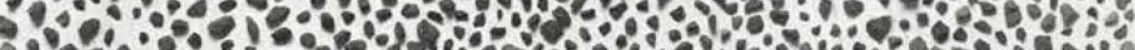

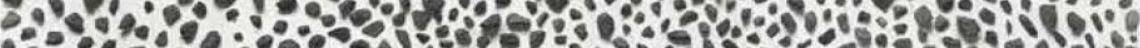
:

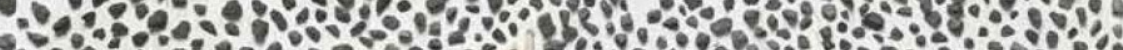

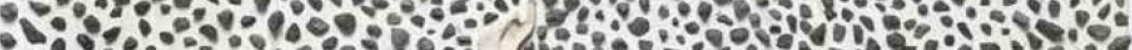
2. -

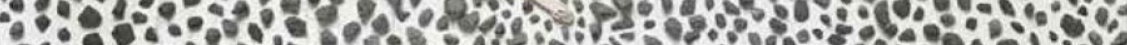

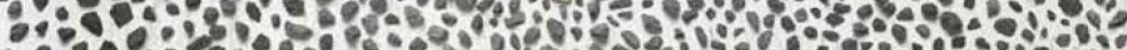

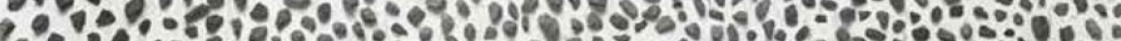

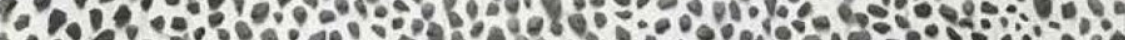

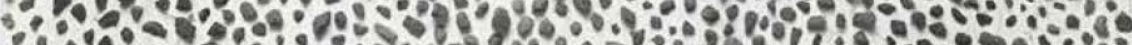

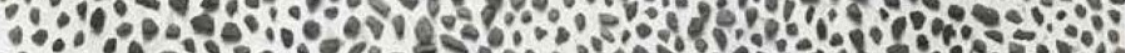
$\rightarrow$ a C. $0.0 .0 \%$.

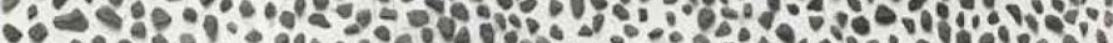

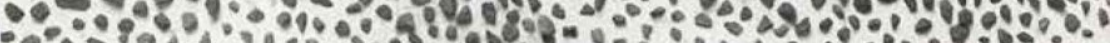
\%,

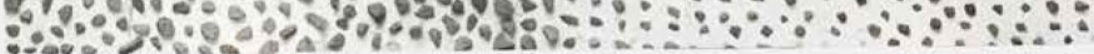




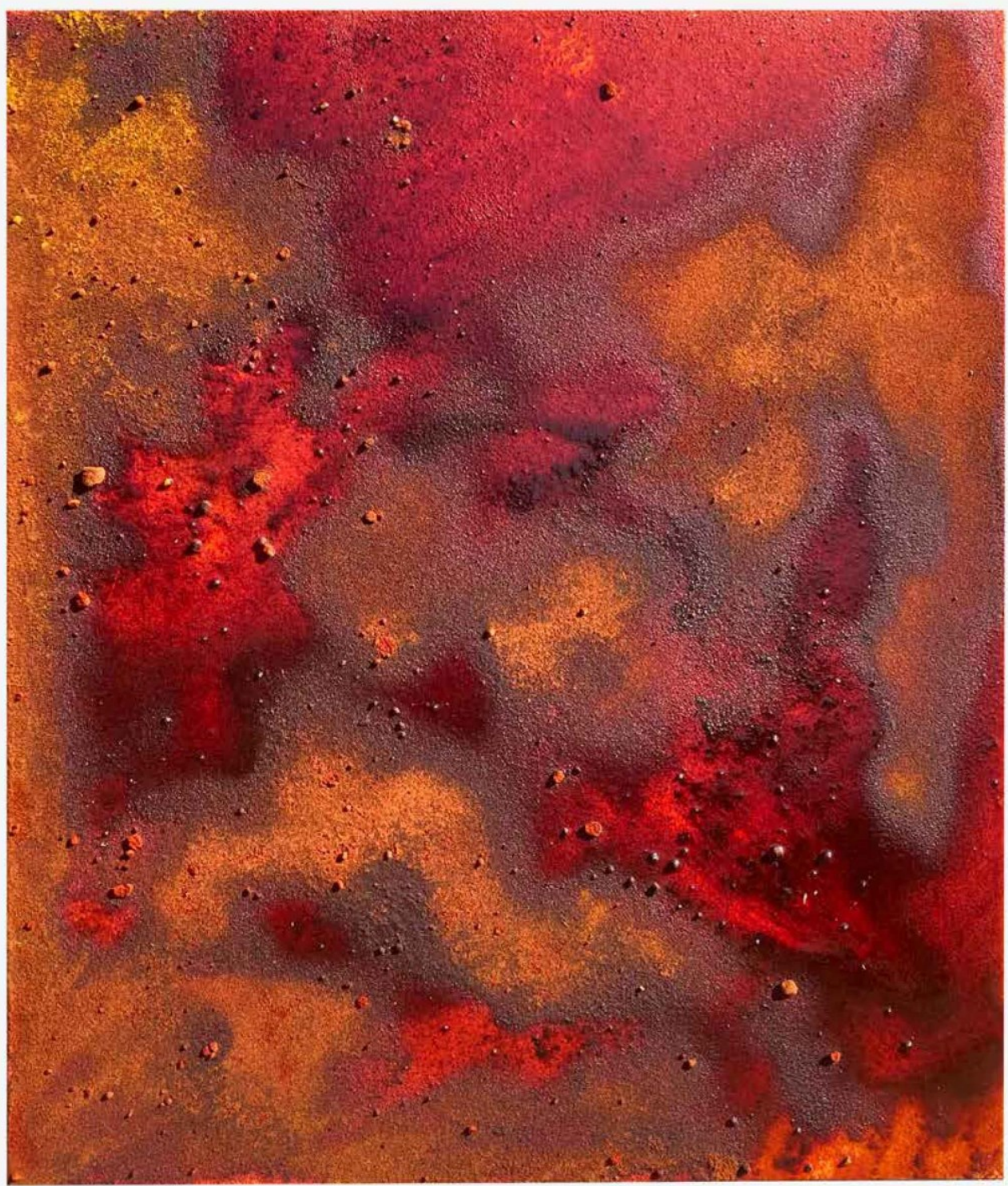

